responses to auditory and tactile stimuli are reported from Bambino Gesu Children's Hospital, Rome, Italy. An excessive startle response caused symmetric jumping of limbs, arms more than legs. Spontaneous attacks developed in 4, particularly in sleep. A family history of epilepsy or febrile convulsions was elicited in 5. Remissions occurred in 4-14 months, spontaneously or in response to valproate. EEGs showed short generalized spike- or polyspike-and-wave discharges. (Ricci S et al. Reflex myoclonic epilepsy in infancy: A new age-dependent idiopathic epileptic syndrome related to startle reaction. Epilepsia April 1995;36:342-348). (Reprints: Dr S Ricci, Section of Neurophysiology, Bambino Gesu Children's Hospital, IRCCS, Piazza S Onofrio 4, 00165 Rome, Italy).

COMMENT. In addition to the reflex nature of these attacks, the abnormal EEG appears to distinguish this syndrome from benign myoclonic epilepsy in infancy. Therapy with anticonvulsants seemed justified when attacks occurred spontaneously and frequently, from 5 to 20 times daily.

\title{
READING EPILEPSY
}

The electroclinical manifestations and natural history of reading epilepsy (RE) in 20 patients diagnosed between 1949 and 1989 are reported from the Mayo Clinic, Rochester, Minnesota. Age at onset ranged from 10 to 46 years (median 17 years). Juvenile myoclonic epilepsy occurred in 4 , and a positive family history for epilepsy in 4, with RE in 1 . Seizures were myoclonic, involving orofacial and jaw muscles, and the upper limbs also in 5 . Generalized tonic-clonic seizures occurred at least once in 16. The EEG showed generalized spike or spike-and wave discharges in 15 cases and left hemisphere discharges in 5. RE was persistent into late adult life but not progressive; it responded to valproic acid. Higher cognitive processes acting as trigger mechanisms other than reading included calculation in 6 , speaking under stress in 5, writing in 2, and playing chess in 1. (Radhakrishnan K, Silbert PL, Klass DW. Reading epilepsy. An appraisal of 20 patients diagnosed at the Mayo Clinic, Rochester, Minnesota, between 1949 and 1989, and delineation of the epileptic syndrome. Brain Feb 1995;118:75-89). (Respond: Donald W Klass MD, Section of Electroencephalography, Mayo Clinic, 200 First Street SW, Rochester, MN 55905).

COMMENT. The authors dedicate their article to Dr Reginald G Bickford on his 81st birthday and we add our congratulations! Bickford (1954) and Bickford, Klass et al (1956) first described the syndrome of reading epilepsy and stressed the importance of precipitating factors in the mechanism of seizures and EEG epileptiform discharges in general. Christie S (1988) found a combination of factors involved in the precipitation of reading epilepsy: saccadic eye movements, articulation, and difficulty of linguistic content. Bickford had alluded to the degree of difficulty of reading matter in his original article. (See Progress in Pediatric Neurology I, 1991, p45).

\section{PREVENTION OF EPILEPSY AND COMPLICATIONS}

Prevention of epilepsy and its consequencies is discussed in a special article from the Department of Neurology, School of Medicine, University of Virginia, Charlottesville. Prevention should apply at various levels: 1 . epileptogenesis may be prevented by a) avoidance of premature birth, in 